\title{
Why are eligible patients not prescribed aspirin in primary care? A qualitative study indicating measures for improvement Duncan Short*1, Martin Frischer ${ }^{1}$, James Bashford ${ }^{1}$ and Darren Ashcroft ${ }^{2}$
}

\author{
Address: ${ }^{1}$ Department of Medicines Management, University of Keele, Keele, Staffordshire, UK, ST5 5BG and ${ }^{2}$ School of Pharmacy and \\ Pharmaceutical Sciences, University of Manchester, Oxford Road, Manchester, UK, M13 9PL \\ Email: Duncan Short* - d.short@keele.ac.uk; Martin Frischer - m.frischer@keele.ac.uk; James Bashford - j.n.r.bashford@keele.ac.uk; \\ Darren Ashcroft - darren.ashcroft@man.ac.uk \\ * Corresponding author
}

Published: 18 July 2003

BMC Family Practice 2003, 4:9
Received: 06 March 2003

Accepted: 18 July 2003

This article is available from: http://www.biomedcentral.com/I47/-2296/4/9

(C) 2003 Short et al; licensee BioMed Central Ltd. This is an Open Access article: verbatim copying and redistribution of this article are permitted in all media for any purpose, provided this notice is preserved along with the article's original URL.

\begin{abstract}
Background: Despite evidence-based guidelines, aspirin prescribing for the secondary prevention of stroke is sub-optimal. Little is known about why general practitioners do not prescribe aspirin to indicated patients. We sought to identify and describe factors that lead general practitioners (GPs) not to prescribe aspirin to eligible stroke patients. This was the first stage of a study exploring the need for and means of improving levels of appropriate aspirin prescribing.
\end{abstract}

Method: Qualitative interviews with I5 GPs in the West Midlands.

Results: Initially, many GPs did not regard their prescribing as difficult or sub-optimal. However on reflection, they gave several reasons that lead to them not prescribing aspirin for eligible patients or being uncertain. These include: difficulties in applying generic guidelines to individuals presenting in consultations, patient resistance to taking aspirin, the prioritisation of other issues in a time constrained consultation and problems in reviewing the medication of existing stroke patients.

Conclusion: In order to improve levels of appropriate aspirin prescribing, the nature and presentation risk information available to GPs and patients must be improved. GPs need support in assessing the risks and benefits of prescribing for patients with combinations of complicating risk factors, while means of facilitating improved GP-patient dialogue are required to help address patient uncertainty. A decision analysis based support system is one option. Decision analysis could synthesise current evidence and identify risk data for a range of patient profiles commonly presenting in primary care. These data could then be incorporated into a user-friendly computerised decision support system to help facilitate improved GP-patient communication. Measures of optimum prescribing based upon aggregated prescribing data must be interpreted with caution. It is not possible to assess whether low levels of prescribing reflect appropriate or inappropriate use of aspirin in specific patients where concordance between the GP and the patient is practised.

\section{Background}

Aspirin is an internationally recognised cost-effective treatment for patients with vascular disease [1]. Prescrib- ing in general practice however has been consistently reported to be sub-optimal despite evidence that the benefits of therapy significantly outweigh the risks for the 
majority of patients [2-4]. Guidelines have been published to assist practitioners, but many patients for whom aspirin may be beneficial do not receive therapy [5-7]. Improving appropriate prescribing of aspirin is a health service priority [2].

The gap between evidence and practice has increasingly been the focus of research. Several reasons have been cited to explain why some general practitioners do not implement evidence based guidelines. These include difficulties in reconciling generic evidence and trial data to individual patients, concerns that decisions may jeopardise the doctor-patient relationship, poor adherence to practice protocols and the absence of computerised systems [8-11].

It is not known whether these reasons cited for other therapeutic areas can explain the decision not to prescribe aspirin in eligible patients. The first stage of a process to improve the implementation of the evidence is therefore to identify the reasons for behaviour and the factors likely to influence any change [12].

This paper reports a qualitative study of GPs to explore why there continues to be a gap between prescribing goals and prescribing practice among patients eligible for aspirin. We focussed upon the secondary prevention of stroke where aspirin is first line therapy yet estimations of use are as low as 39\% [3]. A previous quantitative study concluded that there is "stated unwillingness to advise aspirin prophylaxis by some general practitioners, even when it is clearly indicated" [4]. The objectives of this study were to enhance understanding of aspirin prescribing in the secondary prevention of stroke by exploring difficulties GPs have in deciding whether or not to prescribe for eligible patients and the reasons that they give for not prescribing.

\section{Method}

After approval from the local research ethics committee, fifteen GPs from nine practices in the West Midlands region agreed to take part in the study. The practices were part of a research network established by the Department of Medicines Management, Keele University and varied in size, type of location and catchment area. All forty-five GPs from the network were invited to participate.

As this was a qualitative study, respondents do not constitute a representative sample, but their accounts illustrate a range of experiences and views on prescribing aspirin for patients in general practice. Respondents varied in age, sex and length of time that they had been qualified as a GP. Table 1 shows the characteristics of the interviewees. The sample size was sufficient to reach theoretical saturation.

All interviews lasted between 25 and 35 minutes and were conducted by DS in GP practices. These were based upon a topic guide developed by the authors that was informed by a review of published studies and a pilot interview with a GP. The schedule comprised questions exploring: initial reactions to whether there are difficulties in prescribing aspirin; opinions of published evidence suggesting suboptimal prescribing; the ease of implementing national guidelines; examples of GPs' own prescribing uncertainty or difficulties.

All interviews were tape-recorded and transcribed verbatim with the consent of each participant. Transcripts were analysed for major themes through an iterative process of comparison and evaluation. Each were revisited and revised as further data was gathered. DS led on analysis and met with MF and JB regularly to discuss shared transcripts. Emergent themes were examined and raised in subsequent interviews with practitioners $[13,14]$.

\section{Results}

The interviews identified a range of experiences and views concerning the prescribing of aspirin for the secondary prevention of stroke. GPs' initial reactions contrasted with thoughts regarding prescribing once the area was explored in more detail; in particular, in scenarios in which GPs described experiencing difficulties or uncertainty in making judgements and decisions.

\section{'Aspirin - what problem?' - Initial reactions of GPs}

The immediate reaction of most GPs when asked whether aspirin prescribing presents a difficulty was that it is relatively straightforward. Practitioners expressed great confidence in their own decision making, seemed very clear about contraindications for prescribing and said it was a straightforward recommendation for most patients.

"I can't understand why GPs aren't using it by the bucketful." GP08

"...for the vast majority, it's quite an easy decision. You do it if there are no obvious contraindications." GP13

All of the GPs were nevertheless acutely aware of the evidence highlighting suboptimal prescribing. Many acknowledged that some patients do not receive aspirin who would benefit from therapy, but opinions about such prescribing varied. Some showed some empathy with the difficulties experienced by GPs, while others were less understanding.

"Some clinicians feel that they understand the issues but it is fairly obvious that there is a significant body that don't." GP01 
Table I: Characteristics of GPs interviewed

\begin{tabular}{ll}
\hline Total number interviewed & $\mathrm{n}=15$ \\
\hline Gender & \\
Male & $\mathrm{n}=13$ \\
Female & $\mathrm{n}=2$ \\
Age (years) & Mean 45 Range 34-59 \\
GP Principals & $\mathrm{n}=15$ \\
Number of years practising & Mean $=16$; range 6-34 \\
Number of partners in their practice & Mean $=4$ Range I-8 \\
Number recording consultation notes on their computer & $\mathrm{n}=15$ \\
\hline
\end{tabular}

"GPs have been shown to continue to struggle ... but my reading of the recommendations is that they are quite straightforward." GP02

As each interview progressed, however, prescribing decisions were revealed to be much more complicated than they may have appeared on the surface. Exploration of difficult situations highlighted that decision-making was less clear than initially suggested and that the issue had at times presented difficulties for all practitioners. Prescribing uncertainty and reasons for indicated patients not being prescribed aspirin were detailed.

\section{Difficulties in prescribing aspirin}

Difficulties with aspirin prescribing could be broadly categorised into three areas: Those arising from (a) clinical uncertainty (b) patient resistance (c) other considerations.

\section{(a) Clinical uncertainty}

All of the practitioners said that decision making regarding aspirin was not as straightforward as guidelines may imply. Many stated that there were numerous occasions when they found it difficult to apply generic guidelines to individual patients.

" [Aspirin guidelines] tend to be fairly simple and linear. But the kind of situations we are in are messy and unpredictable and complicated...it's difficult to work out what you should do." GP14

"... for the individual, a guideline cannot indicate what is right .... they are just not specific." GP05

Several factors inhibiting the application of guidelines were frequently cited:

\section{(i) Complexity of individual cases}

Certain patient characteristics introduced an element of uncertainty about recommending aspirin because of how this affected the balance of risks and benefits for that individual. For example:

- Patients with a history of GI disease, such as dyspepsia or ulcers.

"What do you do with people who have previous GI bleeds? ... Those are big decisions that put you off. ...I would need a lot of confidence to say that that was still the right decision." GP03

- The 'very elderly'.

"... because of their risk of GI disease" GP04

- Patients taking non-steroidal anti-inflammatory drugs.

"Certainly patients on anti-inflammatories it is a difficult decision. ...You have some feel for what sort of effect [on the risk of a bleed] an anti-inflammatory will have but you can't quantify it." GP04

\section{(ii) Incomplete patient information}

Another area that presented difficulty for GPs was where there was uncertainty concerning the information that they had available to make their decision. This may be because of incomplete patient records or if a patient's recall of prior events conflicts with their notes.

"...they say they have vomited blood in the past and you are not quite sure - it is not documented elsewhere. Sometimes looking for corroboration in the records is difficult." GP14

\section{(iii) Aetiology of the initial stroke}

A further difficulty described by GPs was when the aetiology of the initial stroke is unknown. Aspirin should not be prescribed to patients whose first stroke is haemorrhagic and ideally GPs would have this information from a computerised tomography (CT) scan. However, many patients may not undergo such a procedure. 
"...We see patients having TIAs or perhaps minor strokes and we are not referring them...they are not going to get a scan." GP14

This uncertainty about the nature of the stroke is problematic and several said that it increased their anxiety about recommending aspirin. Several said that in such circumstances, based upon probability, they assumed the first event was ischaemic rather than haemorrhagic.

"...what I do in practice is assume that it is a non-haemorrhagic stroke and prescribe but I'm very uneasy about that." GP02

"Obviously in the ideal world you scan them all and you get the answer, but that just isn't feasible. As I understand it, statistically only $15 \%$ of strokes are haemorrhagic so the chances are it isn't that." GP15

One GP described a case where they had considered but not prescribed aspirin:

"... in the back of my mind is the balance between haemorrhagic strokes and thrombotic strokes. ... I can well remember the day when I had a [stroke] patient and said 'really you ought to be on aspirin', and the guy said 'I'm going to go away and think about it.' He was in his forties, and he walked out of the door of the surgery and fifteen minutes later he collapsed with a haemorrhagic stroke and survived. I remember thinking if I had stuck that guy on aspirin he would be dead. And that lurks in the back of my mind... You know that you are going to make some patients more likely to have haemorrhagic events". GP13

This practitioner said that the incident had a powerful influence on their future decision making concerning aspirin.

\section{(b) Prescribing difficulties arising from patients' reluctance to take aspirin}

Patients' reluctance to taking aspirin was also identified as a key factor contributing to the non-prescribing of aspirin to indicated patients. In such cases, practitioners said that if they felt aspirin would be beneficial to the patient then they would usually try and encourage them to take this but that some still chose not to take it.

Reluctant patients described by GPs tended to fall into one of three categories:

(i) Patients haunted by the "ghosts of doctors past"

Some patients were said to be determined to adhere to a previous clinician's instruction 'never' to take aspirin again, regardless of their current GP's advice.
"A lot of patients are stuck in the frame that 'my doctor 20 years ago said I should never take aspirin'. And if you've been in with a GI bleed and your consultant said never take aspirin, then that becomes a very ingrained message ... people often do not recognise that information changes with time, and that messages from somebody who saved their life 20 years ago may not be right now." GP03.

\section{(ii) Patients with knowledge of bad experiences with aspirin}

Further patient resistance can be attributed to negative experiences associated with aspirin for themselves, family or friends.

"If you are a patient and you have vomited blood it's a pretty scary experience and most of them wouldn't want to go through it again" GP03

"I have one old lady who won't take it because one of her relatives committed suicide with aspirin. There is no way you can make her take aspirin ... nothing will change that." GP12

\section{(iii) Patients beliefs}

Some patients were said to have beliefs about aspirin that the GPs viewed as irrational. Practitioners said that it was very difficult to reassure these individuals regarding aspirin and overcome this mindset.

"...some people who have asthma are reluctant to take anything 'aspiriny'.... whether they have got an allergy or not." GP10

Overall, communicating the benefits and risks of the decision to patients was an issue for many GPs. They reported cases where they felt the benefits of aspirin significantly outweighed the risks, but patients were reluctant to take the drug and they did not feel that they could sufficiently reassure them of their recommendation.

\section{(c) Other considerations contributing to non-prescribing for eligible patients}

Several other difficulties for GPs were identified by interviewees that may contribute to indicated patients not receiving prophylactic aspirin:

\section{(i) GPs' perceptions of the benefits of aspirin}

Uncertainty among some GPs regarding the evidence for aspirin was perceived as a barrier to optimum prescribing:

" People have taken a long time to be able to work out what the evidence really is." GP14

"I think we underestimate the benefits of something that seems so simple. It's just aspirin. It's not expensive, it's not new, it's not anything else. It's just aspirin. ... you're 
looking at a patient, looking at hypertensive treatment, looking at lipids and then suddenly think 'ooh aspirin' ...we maybe underestimate its importance." GP03

(ii) Problems in reviewing aspirin use among existing stroke patients GPs described problems in reviewing the medication of long-standing patients. For example, patients may not be seen in routine consultations and therefore reviews may not be carried out.

"We have a burden of people who have already had strokes, that we are not necessarily revisiting ... we may not see them from one month to another and we therefore don't review the decision as to whether they are on aspirin or not." GP03

"The foremost problem is that the doctor and the patient don't meet for whatever reason." GP13

Furthermore, even if patients do present in primary care, they may still not be identified as potentially in need of aspirin.

"I think far more of a problem with prescribing is not thinking about it in the first place or not having a set system in place where the system tags up 'why is this patient not on aspirin?"' GP12

Variations in the computerisation of practices was one explanation suggested for problems in identifying patients for review.

"...if you can produce a report showing that only $10 \%$ are on aspirin then straight away it stirs you into action, but if you can't do that, ...you are not going to do anything.." GP07

\section{(iii) Practical difficulties}

Many GPs described the nature of a time-constrained consultation as not conducive to optimum prescribing. For example, for new stroke patients the GP may have higher priority issues than prophylaxis to address such as mobility and wider stroke management issues.

"The priorities on discharge may be ... 'are they mobile?', 'are they continent?', 'are they in a second floor flat?' and all those sorts of issues probably come to the fore, not 'are they taking aspirin?"' GP03

A further practical difficulty described was that the consultation agenda may be dictated by the patient's immediate practical needs.

"... sometimes for elderly people presenting after a stroke, ... future prevention, may not be their priority... you only have 10 minutes for your patient ... and they might be much more worried about a handrail to get up and down the stairs." GP14

"The patient may come and present an agenda around ... arthritis, depression, whatever, and so within a consultation we may have other things going on other than something that is effectively preventative medicine. And you have to concentrate upon the patient's agenda." GP13

Given the time pressures of primary care consultations, such issues can lead to aspirin not being considered.

\section{(iv) The benefits of prophylaxis for very ill patients}

GPs also reported uncertainty about the benefits aspirin prophylaxis when considering the decision for patients with extremely poor health and poor prognosis. In some cases GPs said that they feel that patients may experience longer life if they took aspirin, but that this may not be in the patient's interests because of their very limited quality of life.

"you may feel that the sort of benefits they are likely to get are not that great because the quality of their life is pretty dismal." GP10

\section{(v) Fear of being challenged by stroke patients}

A lesser theme was that some GPs expressed anxiety about patients' reactions to starting them on aspirin for the first time, if their initial stroke was a long time ago. The potential for patients to challenge the GP because of their time without therapy was an issue.

"It is easy to fall through the net. Several times I have done it myself ... I mean a chap came in, ... said 'why haven't I had aspirin before?' ... I had seen him so many times I just hadn't got round to doing it. ... he should have been on it." GP12

\section{Discussion}

Following the publication of the National Service Framework (NSF) for Coronary Heart Disease in 2000 it is a priority to improve levels of appropriate aspirin prescribing. This study has provided fresh insight into the difficulties facing general practitioners. A range of reasons why practitioners may not prescribe aspirin to eligible patients have been identified, which include reservations resulting from clinical uncertainty, patients' reluctance to taking aspirin, perceived benefits of treatment, and issues relating to pragmatism. In practice, these issues have important implications if prescribing targets are used to monitor the quality of care provided to patients.

One major theme to emerge was the difficulties that practitioners have in applying general guideline 
recommendations to individual patients presenting with a personal set of histories and risks. Welton and colleagues showed quantitatively that many GPs responded to complex cases by not prescribing and our study has provided qualitative detail to reinforce this belief [4]. Every practitioner described patients with whom they had struggled to apply the guideline as other factors had complicated their judgement and led them to question whether the benefits outweigh the risks. Simple reinforcement of existing guidelines cannot resolve this uncertainty. There is a need to develop more detailed guidance closer to individual patients' characteristics or target additional education based upon real life cases to clarify risks and benefits for prescribers faced with patients of particular risk profiles. Decision analytical modelling is one potential means of addressing this. Decision analysis is a means of synthesising current evidence and models could be pre-calculated for a number of patient profiles commonly presenting in primary care. The results could then be incorporated into a support system for GPs to access in a consultation.

Patient reluctance to take aspirin was also reported to be an important barrier to prescribing. Even when GPs are confident of the benefits of aspirin, patients may still decide that they would prefer not to receive therapy. Such patient reservations have been identified in other disease contexts $[15,16]$. Many general practitioners who were interviewed often felt unable to adequately communicate the reason for their recommendation to patients who expressed concerns. Current guidance gives insufficient weight to the patient's perspective of risks and improved means of facilitating improved GP-patient dialogue around aspirin therapy is required. Decision support systems have been shown to increase patients' knowledge and agreement between patients' values and decisions, and is one potential solution [17].

It is however important to recognise that a concordant consultation about aspirin may result in an informed patient declining therapy and that this is acceptable [18]. In striving to meet the needs of the NSF, GPs may inappropriately feel that they have failed if the patient does not accept treatment. If we accept that informed patients can choose not to receive therapy, then care is required when using aggregated aspirin prescribing data as a measure of optimum prescribing. In the absence of individual details, it is not possible to be sure whether low levels of prescribing reflect appropriate or inappropriate use of aspirin in specific patients. Individual circumstances for each decision and patient choice are key determinants of whether or not patients receive treatment and in a healthcare system that encourages concordance between the GP and the patient, such a measure may be an unreliable indicator of prescribing quality. If the new GP contract is implemented there may be the facility to record in prescribing data the patients who make informed decisions not to accept a medical recommendation. Current aggregated data however must nevertheless be interpreted with caution.

\section{Conclusion}

In order to improve the appropriate prescribing of aspirin for eligible patients, further research should examine both GPs' and patients' information needs. A failure to adequately support practitioners' decision-making or ensure that this can be effectively communicated will inevitably lead to uncertainty and misunderstandings about the risks and benefits of treatment for individual patients. Support systems underpinned by evidence-based decision analytical models of patient profiles are one potential means of assisting many practitioners in their decision making.

Furthermore, measures of suboptimal prescribing based upon aggregate prescribing data should be interpreted with caution in the absence of more detailed assessment of issues concerning the individual patient.

\section{List of abbreviations}

GP - General Practitioner

CT scan - Computerised tomography scan.

NSF - National Service Framework

\section{Competing interests}

None declared.

\section{Authors' contributions}

DS conducted all interviews. DS, MF and JB were involved in the analysis of the data. All authors contributed in the design of the study and in the interpretation of the results. All authors were involved in constructing the manuscript and have read and approved the final version.

\section{Acknowledgements}

We are grateful to the general practitioners that participated in the study for their time and openness in discussing the issues. We are also grateful to Professor Alison Blenkinsopp for her comments on a draft of this paper.

\section{References}

I. Antiplatelet Trialists' Collaboration: Collaborative overview of randomised trials of antiplatelet therapy-I: Prevention of death, myocardial infarction, and stroke by prolonged antiplatelet therapy in various categories of patients $B M J$ 1994, 308:81-106.

2. Department of Health National Service Framework ('coronary heart disease'): Department of Health. London 2000 [http:// www.doh.gov.uk/nsf/nsfhome.htm].

3. McCallum AK, Whincup PH, Morris RW, Thomson A and Walker M: Aspirin use in middle-aged men with cardiovascular disease: are opportunities being missed? British Journal of General Practice 1997, 47:4|7-42I.

4. Welton M, Croft $P$ and Welton J: General practitioners' use of aspirin in the secondary prevention of vascular events: 
knowledge, attitudes, and current practice British Journal of General Practice 1999, 49:607-610.

5. Royal College of Physicians: National Clinical Guidelines for Stroke [http://www.rcplondon.ac.uk/pubs/books/stroke/index.htm]. 6. Scottish Intercollegiate Guidelines Network (SIGN): "Antithrombotic Therapy. A national clinical guideline SIGN publications 1999:36.

7. Scottish Intercollegiate Guidelines Network (SIGN): "Management of patients with stroke I: Assessment, Investigation, Immediate Management and Secondary Prevention. A national clinical guideline recommended for use in Scotland." SIGN publications 1997.

8. Sweeney K: How can evidence-based medicine help patients in general practice? Fam Pract 1996, I3(6):489-90.

9. Freeman $A C$ and Sweeney K: Why general practitioners do not implement evidence: qualitative study BMJ 200I, 323(732I): $1100-2$.

10. Cranney M, Warren E, Barton S, Gardner K and Walley T: Why do GPs not implement evidence-based guidelines? A descriptive study Fam Pract 200I, 18(4):359-63.

1I. Summerskill WS and Pope C: 'I saw the panic rise in her eyes, and evidence-based medicine went out of the door.' An exploratory qualitative study of the barriers to secondary prevention in the management of coronary heart disease Fam Pract 2002, 19(6):605-10.

12. NHS Centre for Reviews and dissemination, University of York. Effective Health Care Bulletin Getting Evidence into Practice 1999, 5:1.

13. Gilbert N: Researching Social Life Sage Publications London 1995.

14. Miles MB and Huberman AM: Qualitative data analysis: an expanded sourcebook London: Sage 1994.

15. Donovan JI and Blake DR: Patient non-compliance: deviance or reasoned decision-making? Soc Sci Med 1992, 34:507-5I3.

16. Benson J and Britten N: Patients' decisions about whether or not to take antihypertensive drugs: qualitative study $B M J$ 2002, 325:873-876.

17. Molenaar S, Sprangers MA, Postma-Schuit FC, Rutgers EJ, Noorlander J, Hendriks J and de Haes HC: Feasibility and effects of decision aids Med Decis Making 2000, 20(I): I I 2-27.

18. Report of the Royal Pharmaceutical Society of Great Britain. From Compliance to Concordance: Achieving Shared Goals in Medicine Taking 1997.

\section{Pre-publication history}

The pre-publication history for this paper can be accessed here:

http://www.biomedcentral.com/1471-2296/4/9/prepub
Publish with Biomed Central and every scientist can read your work free of charge

"BioMed Central will be the most significant development for disseminating the results of biomedical research in our lifetime. "

Sir Paul Nurse, Cancer Research UK

Your research papers will be:

- available free of charge to the entire biomedical community

- peer reviewed and published immediately upon acceptance

- cited in PubMed and archived on PubMed Central

- yours - you keep the copyright
BioMedcentral 